\title{
Cost-Benefit Analysis of a Computer System with Priority to Preventive Maintenance over Hardware Repair Subject to Maximum Operation and Repair Times
}

\author{
A. K. Barak ${ }^{1 *}$ and S. C. Malik ${ }^{2}$ \\ Department of Statistics, M.D. University, Rohtak-124001, Haryana, India \\ ashishbarak2020@gmail.com',sc_malik@rediffmail.com²
}

\begin{abstract}
A computer system is analyzed considering the concept of priority to Preventive Maintenance (PM) over hardware repair subject to maximum operation and repair times. Two-identical units of the computer system have been taken up-one is initially operative and other is kept as spare in cold standby. In each unit, hardware $(\mathrm{h} / \mathrm{w})$ and software $(\mathrm{s} / \mathrm{w})$ work together and may fail independently from normal mode. There is a single server who visits the system immediately when needed to carry out repair activities. PM of the unit is conducted after a Maximum Operation Time (MOT). If repair of the $\mathrm{h} / \mathrm{w}$ is not possible up to a maximum repair time, it is replaced by a new one with some replacement time. However, only replacement of the $s / w$ is made giving some replacement time with the new one when s/w fails to execute the program properly. Priority to PM of the unit is given over $\mathrm{h} / \mathrm{w}$ repair. The failure time of the unit follows negative exponential distribution while the distributions of PM, repair and replacements are taken as arbitrary. To carry out the cost-benefit analysis, expressions for several reliability measures are obtained by using semi-Markov and regenerative point technique. The graphical behaviour of some important performance measures of the system has been examined giving particular values to various parameters and costs.
\end{abstract}

Keywords: Computer System, h/w and s/w Failure, Priority, Maximum Operation and Repair Time, Preventive Maintenance and Cost-Benefit Analysis.

2000 Mathematics Subject Classification: $90 B 25$ and $60 K 10$

\section{Introduction}

In spite of increasing development and availability of new computer technologies, little work has been dedicated to the cost-benefit analysis of the computer systems with independent failure of $h / w$ and $\mathrm{s} / \mathrm{w}$ components. Also, most of the research work in the area of $\mathrm{h} / \mathrm{w}$ and $\mathrm{s} / \mathrm{w}$ reliability has been carried out by considering either of $\mathrm{h} / \mathrm{w}$ component or s/w component. Friedman and Tran [1], and Welke et al. [2] tried to establish a combined reliability model for the whole system including both h/w and s/w. Further, it is observed that continued operation and ageing of these systems gradually reduce their performance, reliability and safety. It is also proved that preventive maintenance can slow the deterioration process of a repairable system and restore the system in a younger age or state. Thus, the method of preventive maintenance can be used to improve the reliability and profit of systems.

\footnotetext{
* Corresponding author:
}

A. K. Barak (ashishbarak2020@gmail.com) 
Recently, Malik and Anand [3] developed some reliability models for a computer system introducing the concepts of redundancy and independent failure of $h / w$ and s/w. Furthermore, the reliability of a system can be increased by making replacement of the components by new one in case repair time is too long i.e., if it extends to a pre-specific time. Singh and Agrafiotis [4] analyzed stochastically a two-unit cold standby system subject to maximum operation and repair time. It is a matter of record that, not much work related to the reliability modeling of computer systems with the concepts of PM, MOT and Maximum Repair Time (MRT) has been reported so far in the literature.

In view of the above, the main object of this paper is set to analyze a computer system with the concepts of Redundancy, PM, MOT and MRT. For this, a stochastic model is developed by taking two identical units of a computer system in which one unit is operative and other is kept as cold standby. The hardware and software in the unit work together and may fail independently. A single server visits the system to carry out the repair activities. The unit undergoes for PM after an MOT at normal mode. The priority is given to PM of the system over hardware repair. If repair of the $\mathrm{h} / \mathrm{w}$ is not possible up to a pre-specific time (called Maximum Repair Time), it is replaced by new one with some replacement time. However, only replacement of the software giving some replacement time by new one is made in case, it fails to execute the programs properly.

The random variables are independent and uncorrelated to each other. The switch devices, preventive maintenance and repair are perfect. The failure time $\mathrm{h} / \mathrm{w}$ and s/w are exponentially distributed, while the distributions of PM, repair and replacement times are taken as arbitrary with different probability density functions. The expressions for various reliability measures such as Mean Time to System Failure (MTSF), availability, busy period of the server due to Preventive Maintenance, repair, hardware replacement, software replacement, expected number of software replacements, hardware replacements and expected number of visits of the server are derived by using semi-Markov process and regenerative point . Graphs for the results obtained for a particular case are drawn to depict the behaviour of MTSF, availability and profit of the system model.

\section{Notations}

E

$\mathrm{N}_{\mathrm{o}}$

Cs

$a / b$

$\lambda_{1} / \lambda_{2}$

$\alpha_{0}$

$\beta_{0}$

$\mathrm{Pm} / \mathrm{PM}$

: The set of regenerative states

: The unit is operative and in normal mode

: The unit is in cold standby

: Probability that the system has hardware/software failure

: Constant hardware/software failure rate

: Maximum constant rate of Operation Time

: Maximum constant rate of Repair Time.

: The unit is under Preventive Maintenance/under Preventive Maintenance continuously from previous state

WPm/WPM : The unit is waiting for Preventive Maintenance/waiting for Preventive Maintenance from previous state

HFur/HFUR : The unit is failed due to hardware and is under repair/under repair continuously from previous state

HFurp/HFURP : The unit is failed due to hardware and is under replacement/under replacement continuously from previous state

HFwr / HFWR : The unit is failed due to hardware and is waiting for repair/waiting for repair continuously from previous state

SFurp/SFURP : The unit is failed due to the software and is under replacement/under replacement continuously from previous state

SFwrp/SFWRP : The unit is failed due to the software and is waiting forreplacement/waiting for replacement continuously from previous state

$h(t) / H(t) \quad:$ pdf/cdf of replacement time of unit due to software

$g(t) / G(t) \quad:$ pdf/cdf of repair time of the hardware

$m(t) / M(t) \quad:$ pdf/cdf of replacement time of the hardware 
$f(t) / F(t) \quad:$ pdf/cdf of the time for PM of the unit $q_{i j}(t) / Q_{i j}(t) \quad:$ pdf / cdf of passage time from regenerative state $\mathrm{i}$ to a regenerative state $\mathrm{j}$ or to a failed state $\mathrm{j}$ without visiting any other regenerative state in $(0, \mathrm{t}]$

pdf/cdf : Probabilitydensity function/Cumulative density function

$q_{i j, k r}(t) / Q_{i j, k r}(t):$ pdf/cdf of direct transition time from regenerative state $i$ to a regenerative state $\mathrm{j}$ or to a failed state $\mathrm{j}$ visiting state $\mathrm{k}, \mathrm{r}$ once in $(0, \mathrm{t}]$

$\mu_{i}(t) \quad$ : Probability that the system up initially in state $S_{i} \in E$ is up at time t without visiting to any regenerative state

$W_{i}(t) \quad$ : Probability that the server is busy in the state $S_{i}$ upto time ' $t$ 'without making any transition to any other regenerative state or returning to the same state via one or more non-regenerative states.

$m_{i j} \quad:$ Contribution to mean sojourn time $\left(\mu_{i}\right)$ in state $S_{i}$ when system transit directly to state $S_{j}$ so that $\mu_{i}=\sum_{j} m_{i j}$ and $\mathrm{m}_{i j}=\int t d Q_{i j}(t)=-q_{i j}^{*}{ }^{\prime}(0)$

(S)/C) : Symbol for Laplace-Stieltjes Convolution/Laplace Convolution

$\sim$ / * : Symbol for Laplace-Steiltjes Transform

(LST) / Laplace Transform (LT)

' (desh) : : Used to represent alternative result

Considering these symbols, the following are possible transition states of the system model:

$\mathrm{S}_{0}=\left(\mathrm{N}_{\mathrm{o}}, \mathrm{Cs}\right)$,

$\mathrm{S}_{2}=\left(\mathrm{N}_{\mathrm{o}}, \mathrm{HFur}\right)$,

$\mathrm{S}_{1}=\left(\mathrm{N}_{\mathrm{o}}, \mathrm{Pm}\right)$,

$\mathrm{S}_{4}=\left(\mathrm{N}_{\mathrm{o}}\right.$, HFurp $)$,

$\mathrm{S}_{3}=\left(\mathrm{N}_{\mathrm{o}}\right.$, SFurp $)$,

$\mathrm{S}_{6}=(\mathrm{HFwr}, \mathrm{PM})$,

$\mathrm{S}_{5}=(\mathrm{Pm}, \mathrm{HFwr})$,

$\mathrm{S}_{8}=(\mathrm{PM}, \mathrm{SFwrp})$,

$\mathrm{S}_{7}=($ SFURP, HFwr),

$\mathrm{S}_{9}=($ SFURP, WPm $)$,

$\mathrm{S}_{10}=$ (SFURP, SFwrp),

$\mathrm{S}_{11}=$ (HFUR, SFwrp),

$\mathrm{S}_{12}=($ HFUR, HFwr),

$\mathrm{S}_{13}=(\mathrm{WPm}, \mathrm{PM})$,

$\mathrm{S}_{14}=$ (HFurp, SFwrp),

$\mathrm{S}_{15}=$ (HFWR, HFurp),

$\mathrm{S}_{16}=($ HFURP, WPm),

$\mathrm{S}_{17}=$ (HFURP, SFwrp),

$\mathrm{S}_{18}=($ HFURP, HFwr)

All the transitions states are given on the base of system model given in state transition diagram, Figure 1.

\section{Transition Probabilities and Mean Sojourn Times}

Simple probabilistic considerations yield the following expressions for the non-zero elements

$$
p_{i j}=Q_{i j}(\infty)=\int_{0}^{\infty} q_{i j}(t) d t \text { as }
$$

$p_{01}=\frac{\alpha_{0}}{a \lambda_{1}+b \lambda_{2}+\alpha_{0}}, \quad p_{02}=\frac{a \lambda_{1}}{a \lambda_{1}+b \lambda_{2}+\alpha_{0}}$,

$p_{03}=\frac{b \lambda_{2}}{a \lambda_{1}+b \lambda_{2}+\alpha_{0}}$

$p_{10}=f^{*}\left(a \lambda_{1}+b \lambda_{2}+\alpha_{0}\right), \quad p_{16}=\frac{a \lambda_{1}}{a \lambda_{1}+b \lambda_{2}+\alpha_{0}}\left[1-f^{*}\right.$

$p_{18}=\frac{b \lambda_{2}}{a \lambda_{1}+b \lambda_{2}+\alpha_{0}}\left[1-f^{*}\left(a \lambda_{1}+b \lambda_{2}+\alpha_{0}\right)\right]=p_{13.8}$,

$p_{20}=g^{*}\left(a \lambda_{1}+b \lambda_{2}+\alpha_{0}\right)$

$p_{1.13}=\frac{\alpha_{0}}{a \lambda_{1}+b \lambda_{2}+\alpha_{0}}\left[1-f^{*}\left(a \lambda_{1}+b \lambda_{2}+\alpha_{0}\right)\right]=p_{11.13}$,

$p_{30}=h^{*}\left(a \lambda_{1}+b \lambda_{2}+\alpha_{0}\right)$,

$p_{24}=\frac{\beta_{0}}{a \lambda_{1}+b \lambda_{2}+\alpha_{0}+\beta_{0}}\left[1-g^{*}\left(a \lambda_{1}+b \lambda_{2}+\alpha_{0}+\beta_{0}\right)\right]$,

$p_{40}=m^{*}\left(a \lambda_{1}+b \lambda_{2}+\alpha_{0}\right)$,

$p_{25}=\frac{\alpha_{0}}{a \lambda_{1}+b \lambda_{2}+\alpha_{0}+\beta_{0}}\left[1-g^{*}\left(a \lambda_{1}+b \lambda_{2}+\alpha_{0}+\beta_{0}\right)\right]$,

$p_{52}=f^{*}(0), \quad p_{16.1}=m^{*}(0)$,

$p_{2.11}=\frac{b \lambda_{2}}{a \lambda_{1}+b \lambda_{2}+\alpha_{0}+\beta_{0}}\left[1-g^{*}\left(a \lambda_{1}+b \lambda_{2}+\alpha_{0}+\beta_{0}\right)\right]$,

$p_{62}=f^{*}(0), \quad p_{72}=h^{*}(0)$

$p_{2.12}=\frac{a \lambda_{1}}{a \lambda_{1}+b \lambda_{2}+\alpha_{0}+\beta_{0}}\left[1-g^{*}\left(a \lambda_{1}+b \lambda_{2}+\alpha_{0}+\beta_{0}\right)\right]$,

$p_{83}=f^{*}(0), \quad p_{91}=h^{*}(0)$,

$p_{37}=\frac{a \lambda_{1}}{a \lambda_{1}+b \lambda_{2}+\alpha_{0}}\left[1-h^{*}\left(a \lambda_{1}+b \lambda_{2}+\alpha_{0}\right)\right]=p_{32.7}$,

$p_{10.3}=h^{*}(0), \quad p_{11.3}=g^{*}\left(\beta_{0}\right)$,

$p_{39}=\frac{\alpha_{0}}{a \lambda_{1}+b \lambda_{2}+\alpha_{0}}\left[1-h^{*}\left(a \lambda_{1}+b \lambda_{2}+\alpha_{0}\right)\right]=p_{31.9}$,

$p_{11.14}=1-g^{*}\left(\beta_{0}\right), \quad p_{12.2}=g^{*}\left(\beta_{0}\right)$,

$p_{3,10}=\frac{b \lambda_{2}}{a \lambda_{1}+b \lambda_{2}+\alpha_{0}}\left[1-h^{*}\left(a \lambda_{1}+b \lambda_{2}+\alpha_{0}\right)\right]=p_{33.10}$,

$p_{12.15}=1-g^{*}\left(\beta_{0}\right), \quad p_{13.1}=f^{*}(0)$,

$p_{4.16}=\frac{\alpha_{0}}{a \lambda_{1}+b \lambda_{2}+\alpha_{0}}\left[1-m^{*}\left(a \lambda_{1}+b \lambda_{2}+\alpha_{0}\right)\right]=p_{41.16}$,

$p_{14.3}=m^{*}(0), \quad p_{15.2}=m^{*}(0)$, 


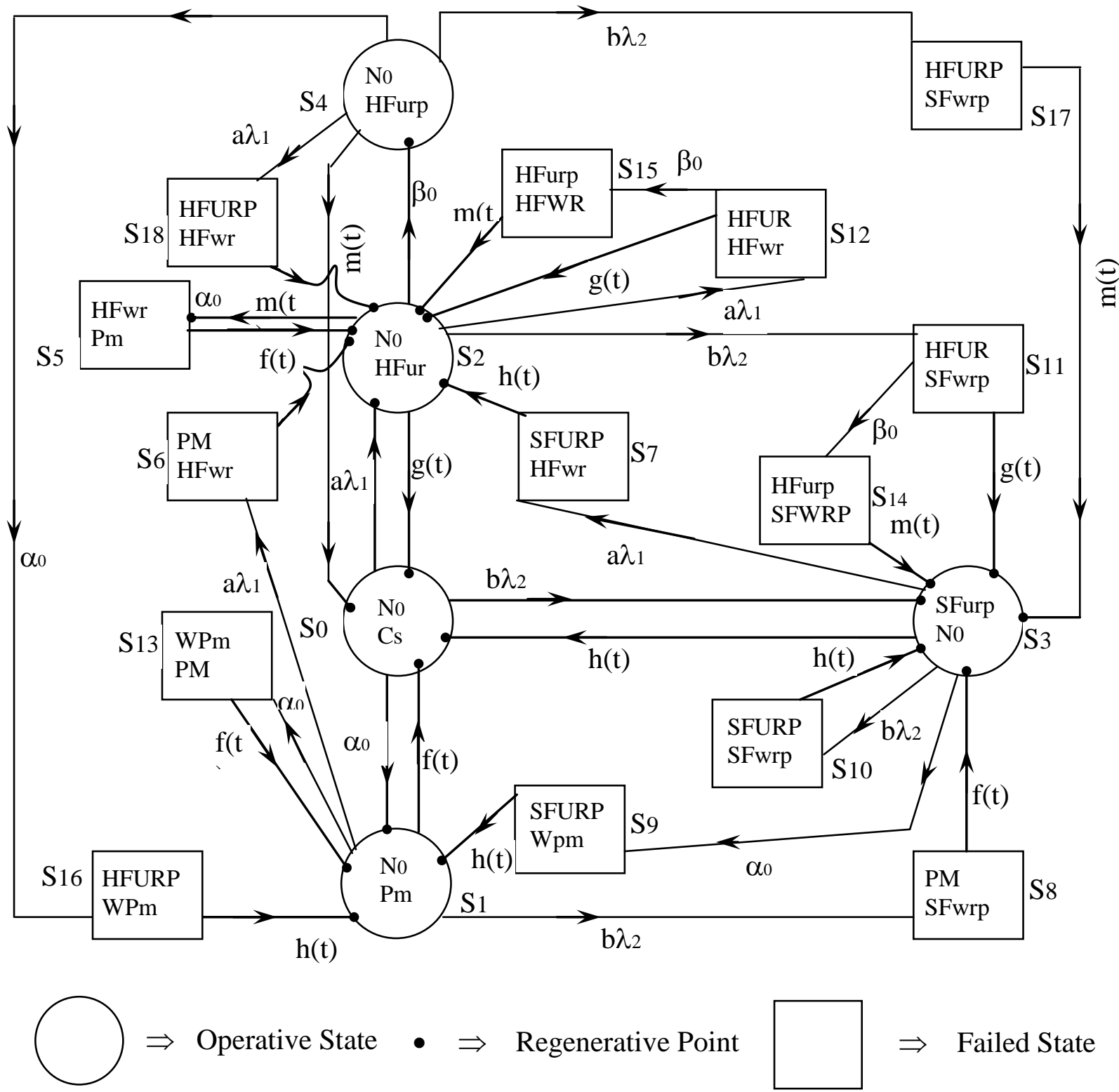

Figure 1. State Transition Diagram.

$$
\begin{aligned}
& p_{4,17}=\frac{b \lambda_{2}}{a \lambda_{1}+b \lambda_{2}+\alpha_{0}}\left[1-m^{*}\left(a \lambda_{1}+b \lambda_{2}+\alpha_{0}\right)\right]=p_{43.17}, \quad p_{22.12}=\frac{a \lambda_{1}}{a \lambda_{1}+b \lambda_{2}+\alpha_{0}+\beta_{0}}\left[1-g^{*}\left(a \lambda_{1}+b \lambda_{2}+\alpha_{0}+\beta_{0}\right)\right] \\
& p_{17.3}=m^{*}(0), \quad p_{18.2}=m^{*}(0), \quad g^{*}\left(\beta_{0}\right) \\
& p_{4.18}=\frac{a \lambda_{1}}{a \lambda_{1}+b \lambda_{2}+\alpha_{0}}\left[1-m^{*}\left(a \lambda_{1}+b \lambda_{2}+\alpha_{0}\right)\right]=p_{42.18}, \quad p_{22.12,15}=\frac{a \lambda_{1}}{a \lambda_{1}+b \lambda_{2}+\alpha_{0}+\beta_{0}}\left[1-g^{*}\left(a \lambda_{1}+b \lambda_{2}+\alpha_{0}+\beta_{0}\right)\right] \\
& p_{23.11}=\frac{b \lambda_{2}}{a \lambda_{1}+b \lambda_{2}+\alpha_{0}+\beta_{0}}\left[1-g^{*}\left(a \lambda_{1}+b \lambda_{2}+\alpha_{0}+\beta_{0}\right)\right] \quad \text { It can be easily verified that } \\
& {\left[g^{*}\left(\beta_{0}\right)\right]} \\
& p_{23.11,14}=\frac{b \lambda_{2}}{a \lambda_{1}+b \lambda_{2}+\alpha_{0}+\beta_{0}}\left[1-g^{*}\left(a \lambda_{1}+b \lambda_{2}+\alpha_{0}+\beta_{0}\right)\right] \\
& p_{01}+p_{02}+p_{03}=p_{10}+p_{16}+p_{18}+p_{1.13}=p_{20}+p_{24}+p_{25} \\
& {\left[1-g^{*}\left(\beta_{0}\right)\right]} \\
& +p_{2.11}+p_{2.12}=p_{30}+p_{37}+p_{39}+p_{3,10}=p_{40}+p_{4.16}+p_{4.17} \\
& +p_{4.18}=p_{5.2}=p_{62}=p_{72}=p_{83}=p_{91}=p_{10.3}=p_{11.3}+p_{11.14} \\
& =p_{12.2}+p_{12.15}=p_{13.1}=p_{14.1}=p_{15.2}=p_{16.1}=p_{17.3}=p_{18.2}
\end{aligned}
$$


$=p_{10}+p_{12.6}+p_{11.13}+p_{13.8}=p_{20}+p_{24}+p_{25}+p_{23,11}+p_{23.11,14}$

$+p_{22,12}+p_{22.12,15}=p_{30}+p_{31.9}+p_{32.7}+p_{33.10}=p_{40}+p_{41.17}$

$+p_{42.19}+p_{43.18}=1$

The mean sojourn times $\left(\mu_{\mathrm{i}}\right)$ is the state $S_{i}$ are

$$
\begin{aligned}
& \mu_{0}=\frac{1}{a \lambda_{1}+b \lambda_{2}+\alpha_{0}}, \quad \mu_{1}=\frac{1}{a \lambda_{1}+b \lambda_{2}+\alpha_{0}+\alpha}, \\
& \mu_{2}=\frac{1}{a \lambda_{1}+b \lambda_{2}+\alpha_{0}+\theta+\beta_{0}}, \\
& \mu_{3}=\frac{1}{a \lambda_{1}+b \lambda_{2}+\alpha_{0}+\beta}, \quad \mu_{4}=\frac{1}{a \lambda_{1}+b \lambda_{2}+\alpha_{0}+\gamma}, \\
& \mu_{1}^{\prime}=\frac{1}{\alpha}, \quad \mu_{3}^{\prime}=\frac{1}{\beta}, \quad \mu_{4}^{\prime}=\frac{1}{\gamma}, \quad \mu_{5}=\frac{1}{\alpha}, \\
& \mu_{2}^{\prime}=\quad \begin{array}{r}
\left(a \lambda_{1}+b \lambda_{2}+\alpha_{0}+\theta+\beta_{0}\right)^{3}\left(\theta+\beta_{0}\right)^{3}+\left(a \lambda_{1}+b \lambda_{2}\right) \\
\left(a \lambda_{1}+b \lambda_{2}+\alpha_{0}+\beta_{0}\right)^{2}\left\{\theta\left(a \lambda_{1}+b \lambda_{2}+\alpha_{0}+\theta+\beta_{0}\right)\right. \\
\left(a \lambda_{1}+b \lambda_{2}+\alpha_{0}+\beta_{0}\right)+\theta\left(a \lambda_{1}+b \lambda_{2}+\alpha_{0}+\theta+\beta_{0}\right) \\
\left.\quad\left(\theta+\beta_{0}\right)-\theta \beta_{0}\left(\theta+\beta_{0}\right)\right\}+\left(a \lambda_{1}+b \lambda_{2}\right)\left(a \lambda_{1}+b \lambda_{2}\right. \\
\left.\quad+\alpha_{0}+\theta+\beta_{0}\right)\left\{\left(a \lambda_{1}+b \lambda_{2}+\alpha_{0}+\beta_{0}\right)^{3}\left(\theta+\beta_{0}\right)^{3}\right.
\end{array} \\
& \quad+\left(a \lambda_{1}+b \lambda_{2}+\alpha_{0}+\beta_{0}\right)^{3}\left(\theta+\beta_{0}\right)^{3}+\left(a \lambda_{1}+b \lambda_{2}\right. \\
& \left.+\alpha_{0}+\beta_{0}\right)^{3}+\left(\theta+\beta_{0}\right)-\theta\left(a \lambda_{1}+b \lambda_{2}+\alpha_{0}+\theta+\beta_{0}\right) \\
& \left.\left(\theta+\beta_{0}\right)\right\}-\alpha_{0}\left(a \lambda_{1}+b \lambda_{2}+\theta+\alpha_{0}+\beta_{0}\right)^{2}\left(\theta+\beta_{0}\right)^{3} \\
& \left(a \lambda_{1}+b \lambda_{2}+\alpha_{0}+\beta_{0}\right)+\theta \alpha_{0}\left(a \lambda_{1}+b \lambda_{2}+\alpha_{0}+\beta_{0}\right) \\
& \quad\left(a \lambda_{1}+b \lambda_{2}+\alpha_{0}+\theta+\beta_{0}\right)\left(\theta+\beta_{0}\right) \\
& \quad-\theta\left(a \lambda_{1}+b \lambda_{2}+\alpha_{0}+\beta_{0}\right)^{2}\left(\theta+\beta_{0}\right)^{2} \alpha_{0}
\end{aligned}
$$

Also,

$$
\begin{aligned}
& m_{01}+m_{02}+m_{03}=\mu_{0} \quad m_{10}+m_{16}+m_{18}+m_{1.13}=\mu_{1} \\
& m_{20}+m_{24}+m_{25}+m_{2.11}+m_{2.12}=\mu_{2} \\
& m_{30}+m_{37}+m_{39}+m_{3.10}+=\mu_{3} \\
& m_{40}+m_{4.16}+m_{4.17}+m_{4.18}+=\mu_{4} \\
& m_{10}+m_{12.6}+m_{13.8}+m_{11.13}=\mu_{1}^{\prime} \text { (say) } \\
& m_{20}+m_{24}+m_{25}+m_{22.12}+m_{22.12,15}+m_{23.11}+m_{23.11,14}=\mu_{2}^{\prime} \text { (say) } \\
& m_{30}+m_{31.9}+m_{32.7}+m_{33.10}=\mu_{3}^{\prime} \text { (say) } \\
& m_{40}+m_{42.18}+m_{43.17}+m_{41.16}=\mu_{4}^{\prime} \text { (say) }
\end{aligned}
$$

\section{Reliability and Mean Time to System Failure (MTSF)}

Let, $\phi_{i}(t)$ be the cdf of first passage time from the regenerative state i to a failed state. Regarding the failed state as absorbing state, we have the following recursive relation for $\phi_{i}(t)$ :

$$
\varphi_{i}(t)=\sum_{j} Q_{i, j}(t) \& \varphi_{i}(t)+\sum_{k} Q_{i^{\prime} k}(t)
$$

Where, $j$ is an un-failed regenerative state to which the given regenerative state $i$ can transit and $k$ is a failed state to which the state $i$ can transit directly. $\tilde{\phi}_{0}(s)$

Taking LT of above relation (6) and solving for We have,

$$
R^{*}(s)=\frac{1-\tilde{\phi}_{0}(s)}{s}
$$

The reliability of the system model can be obtained by taking Laplace inverse transform of (7).

The Mean Time to System Failure (MTSF) is given by

$$
\begin{gathered}
\text { MTSF }=\lim _{s \rightarrow 0} \frac{1-\tilde{\phi}_{0}(s)}{s}=\frac{N_{1}}{D_{1}} \quad \text { where } \\
N_{1}=\mu_{0}+p_{01} \mu_{1}+p_{02} \mu_{2}+p_{03} \mu_{3}+p_{24} p_{02} \mu_{4} \text { and } \\
D_{1}=1-p_{01} p_{10}-p_{02} p_{20}-p_{03} p_{30}-p_{02} p_{24} p_{40}
\end{gathered}
$$

\section{Steady State Availability}

Let, $A_{i}(t)$ be the probability that the system is in up-state at instant ' $t$ ' given that the system entered regenerative state $i$ at $t=0$. The recursive relations for $A_{i}(t)$ are given as

$$
A_{i}(t)=M_{i}(t)+\sum_{j} q_{i, j}^{(n)}(t) @ A_{j}(t)
$$

Where, $j$ is any successive regenerative state to which the regenerative state $i$ can transit through $n \geq 1$ (natural number) transitions. $M_{i}(t)$ is the probability that the system is up initially in state $S_{i} \in E$ is up at time $t$ without visiting to any other regenerative state, we have

$$
\begin{array}{ll}
M_{0}(t)=e^{-\left(a \lambda_{1}+b \lambda_{2}+\alpha_{0}\right) t}, & M_{1}(t)=e^{-\left(a \lambda_{1}+b \lambda_{2}+\alpha_{0}\right) t} \overline{F(t)}, \\
M_{2}(t)=e^{-\left(a \lambda_{1}+b \lambda_{2}+\alpha_{0}+\beta_{0}\right) t} \overline{G(t)} & M_{3}(t)=e^{-\left(a \lambda_{1}+b \lambda_{2}+\alpha_{0}\right) t} \overline{H(t)}, \\
M_{4}(t)=e^{-\left(a \lambda_{1}+b \lambda_{2}+\alpha_{0}\right) t} \overline{M(t)} &
\end{array}
$$

Taking LT of above relations (9) and solving for $A_{0}^{*}(s)$, the steady state availability is given by

$$
A_{0}(\infty)=\lim _{s \rightarrow 0} s A_{0}^{*}(s)=\frac{N_{2}}{D_{2}}
$$

where,

$$
\begin{aligned}
& N_{2}=\left(1-p_{22.12}-p_{22.12,15}-p_{25}-p_{24} p_{42.18}\right)\left[\mu_{0}\left(1-p_{11.13}\right)\right. \\
& \left(1-p_{33.10}\right)-\mu_{0} p_{13.8} p_{31.9}+\mu_{1}\left(1-p_{33.10}\right) p_{01}+\mu_{1} p_{31.9} p_{03}
\end{aligned}
$$


$\left.+\mu_{3} p_{01} p_{13.8}+\mu_{3} p_{03}\left(1-p_{11.13}\right)\right]+\left(p_{23.11}+p_{23.11,14}+p_{24} p_{43.17}\right)$ $\left[-\mu_{0}\left(1-p_{11.13}\right) p_{32.7}-p_{12.6} p_{31.9} \mu_{0}-\mu_{1} p_{01} p_{32.7}+p_{31.9} p_{02} \mu_{1}\right.$ $\left.+p_{01} p_{12.6} \mu_{3}+\left(1-p_{11.13}\right) p_{02} \mu_{3}\right]+\mu_{4} p_{41.16}\left[-\mu_{0} p_{12.6}(1-\right.$ $\left.p_{33.10}\right)-p_{32.7} \mu_{0} p_{13.8}+\mu_{1}\left(1-p_{33.10}\right) p_{02}+p_{32.7} p_{03} \mu_{1}+\mu_{3}$ $\left.p_{02} p_{13.8}-\mu_{3} p_{03} p_{12.6}\right]+\left(\mu_{4} p_{24}+\mu_{2}\right)\left[\left(1-p_{33.10}\right) p_{12.6} p_{01}\right.$ $+p_{01} p_{13.8} p_{32.7}+p_{02}\left(1-p_{11.13}\right)\left(1-p_{33.10}\right)-p_{02} p_{13.8} p_{31.9}$ $\left.+p_{12.6} p_{31.9} p_{03}+p_{03}\left(1-p_{11.13}\right) p_{32.7}\right]$

and

$D_{2}=\left(1-p_{22.12}-p_{22.12,15}-p_{25}-p_{24} p_{42.18}\right)\left[\mu_{0}\left(1-p_{11.13}\right)\right.$ $\left(1-p_{33.10}\right)-\mu_{0} p_{13.8} p_{31.9}+\mu_{1}^{\prime}\left(1-p_{33.10}\right) p_{01}+\mu_{1}^{\prime} p_{31.9} p_{03}+$ $\left.\mu_{3}^{\prime} p_{01} p_{13.8}+\mu_{3}^{\prime} p_{03}\left(1-p_{11.13}\right)\right]+\left(p_{23.11}+p_{23.11,14}+p_{24} p_{43.17}\right)$ $\left[-\mu_{0}\left(1-p_{11.13}\right) p_{32.7}-p_{12.6} p_{31.9} \mu_{0}-\mu_{1}^{\prime} p_{01} p_{32.7}+p_{31.9}\right.$ $\left.p_{02} \mu_{1}^{\prime}+p_{01} p_{12.6} \mu_{3}^{\prime}+\left(1-p_{11.13}\right) p_{02}\right]+p_{24} p_{41.16}\left[-\mu_{0} p_{12.6}\right.$ $\left(1-p_{33.10}\right)-p_{32.7} \mu_{0} p_{13.8}+\mu_{1}^{\prime}\left(1-p_{33.10}\right) p_{02}+p_{32.7} p_{03} \mu_{1}^{\prime}$ $\left.+\mu_{3}^{\prime} p_{02} p_{13.8}-\mu_{3}^{\prime} p_{03} p_{12.6}\right]+\left(\mu_{4}^{\prime} p_{24}+\mu_{2}^{\prime}\right)\left[\left(1-p_{33.10}\right) p_{12.6}\right.$ $p_{01}+p_{01} p_{13.8} p_{32.7}+p_{02}\left(1-p_{11.13}\right)\left(1-p_{33.10}\right)-p_{02} p_{13.8} p_{31.9}$ $\left.+p_{12.6} p_{31.9} p_{03}+p_{03}\left(1-p_{11.13}\right) p_{32.7}\right]$

\section{Busy Period Analysis for Server}

Let, $B_{i}^{P}(t) B_{i}^{R}(t) B_{i}^{S}(t)$ and $B_{i}^{H R p}(t)$ be the probabilities that the server is busy in Preventive maintenance of the system, repairing the unit due to hardware failure, replacement of the software and hardware components at an instant ' $t$ ' given that the system entered state $i$ at $t=0$. The recursive relations for $B_{i}^{P}(t) B_{i}^{R}(t) B_{i}^{S}(t)$ and $B_{i}^{H R p}(t)$ are as follows:

$$
\begin{aligned}
B_{I}^{P}(t) & =W_{i}(t)+\sum_{j} q_{i, j}^{(n)}(t) @ B_{j}^{p}(t) \\
B_{i}^{R}(t) & =W_{i}(t)+\sum_{j} q_{i, j}^{(n)}(t) \subseteq B_{j}^{R}(t) \\
B_{i}^{s}(t) & =W_{i}(t)+\sum_{j} q_{i, j}^{(n)}(t) B_{j}^{s}(t) \\
B_{i}^{H R p}(t) & =W_{i}(t)+\sum_{j} q_{i, j}^{(n)}(t) B_{j}^{H R p}(t)
\end{aligned}
$$

where, $j$ is any successive regenerative state to which the regenerative state $i$ can transit through $n \geq 1$ (natural number) transitions. $W_{i}(t)$ be the probability that the server is busy in state Si due to Preventive Maintenance, hardware and software failure up to time $t$ without making any transition to any other regenerative state or returning to the same via one or more non-regenerative states and so,

$$
\begin{aligned}
W_{1}= & e^{-\left(a \lambda_{1}+b \lambda_{2}+\alpha_{0}\right) t} \bar{F}(t)+\left(\alpha_{0} e^{-\left(a \lambda_{1}+b \lambda_{2}+\alpha_{0}\right) t}(1) \bar{F}(t)\right. \\
& +\left(a \lambda_{1} e^{-\left(a \lambda_{1}+b \lambda_{2}+\alpha_{0}\right) t}(1) \bar{F}(t)\right. \\
& +\left(b \lambda_{2} e^{-\left(a \lambda_{1}+b \lambda_{2}+\alpha_{0}\right) t}(1) \bar{F}(t), \quad W_{5}=\bar{F}(t)\right.
\end{aligned}
$$

$$
\begin{aligned}
& W_{2}=e^{-\left(a \lambda_{1}+b \lambda_{2}+\alpha_{0}+\beta_{0}\right) t} \bar{G}(t)+\left(\alpha_{0} e^{-\left(a \lambda_{1}+b \lambda_{2}+\alpha_{0}+\beta_{0}\right) t}\right) \bar{G}(t) \\
& +\left(a \lambda_{1} e^{-\left(a \lambda_{1}+b \lambda_{2}+\alpha_{0}+\beta_{0}\right) t}(1) \bar{G}(t)\right. \\
& +\left(b \lambda_{2} e^{-\left(a \lambda_{1}+b \lambda_{2}+\alpha_{0}+\beta_{0}\right) t} \odot 1\right) \bar{G}(t) \\
& W_{3}=e^{-\left(a \lambda_{1}+b \lambda_{2}+\alpha_{0}\right) t} \bar{H}(t)+\left(\alpha_{0} e^{-\left(a \lambda_{1}+b \lambda_{2}+\alpha_{0}\right) t} @ 1\right) \bar{H}(t) \\
& +\left(a \lambda_{1} e^{-\left(a \lambda_{1}+b \lambda_{2}+\alpha_{0}\right) t}(1) \bar{H}(t)\right. \\
& +\left(b \lambda_{2} e^{-\left(a \lambda_{1}+b \lambda_{2}+\alpha_{0}\right) t}(1) \bar{H}(t)\right. \\
& +\left(a \lambda_{1} e^{-\left(a \lambda_{1}+b \lambda_{2}+\alpha_{0}\right) t} \subset 1\right) \bar{M}(t) \\
& +\left(b \lambda_{2} e^{-\left(a \lambda_{1}+b \lambda_{2}+\alpha_{0}\right) t}(1) \bar{M}(t)\right.
\end{aligned}
$$

Taking LT of above relations (12) and solving for $B_{i}^{P}(t) B_{i}^{R}(t) B_{i}^{S}(t)$ and $B_{i}^{H R p}(t)$ the time for which server is busy due to $\mathrm{PM}, \mathrm{h} / \mathrm{w}$ repair and $\mathrm{h} / \mathrm{w}$ and s/w replacements respectively is given by

$$
\begin{gathered}
B_{0}^{H}=\lim _{s \rightarrow 0} s B_{0}^{* H}(s)=\frac{N_{3}^{H}}{D_{2}}, \\
B_{0}^{s}=\lim _{s \rightarrow 0} s B_{0}^{* S}(s)=\frac{N_{3}^{s}}{D_{2}}, \\
B_{0}^{R}=\lim _{s \rightarrow 0} s B_{0}^{* R}(s)=\frac{N_{s}^{R}}{D_{2}} \text { and } \\
B_{0}^{H R p}=\lim _{s \rightarrow 0} s B_{0}^{* H R p}(s)=\frac{N_{s}^{H R p}}{D_{2}}
\end{gathered}
$$

where

$$
\begin{aligned}
N_{3}^{p}=(1 & \left.-p_{22.12}-p_{25}-p_{22.12,15} p_{20}-p_{42.18} p_{24}\right) \\
& {\left[\mu_{1}^{\prime}\left(1-p_{33.10}\right) p_{01}+\mu_{1}^{\prime} p_{03} p_{31.9}\right] } \\
& +\left(p_{23.11}+p_{23.11,14}+p_{43.17} p_{24}\right) \\
& {\left[\mu_{1}^{\prime} p_{02} p_{31.9}-\mu_{1}^{\prime} p_{01} p_{32.7}\right]+p_{24} p_{41.16} } \\
& {\left[\mu_{1}^{\prime}\left(1-p_{33.10}\right) p_{02}-\mu_{1}^{\prime} p_{03} p_{32.7}\right]+} \\
& p_{25} \mu_{5}\left[p_{01}\left\{\left(1-p_{33.10}\right) p_{12.6}+p_{32.7} p_{13.8}\right\}\right. \\
& +p_{02}\left\{\left(1-p_{33.10}\right)\left(1-p_{11.13}\right)-p_{31.9} p_{13.8}\right\} \\
& \left.+p_{03}\left\{p_{32.7}\left(1-p_{11.13}\right)+p_{3.19} p_{12.6}\right\}\right] \\
N_{3}^{R}=\mu_{2}^{\prime} & {\left[p_{01}\left\{\left(1-p_{33.10}\right) p_{12.6}+p_{32.7} p_{13.8}\right\}\right.} \\
& +p_{02}\left\{\left(1-p_{33.10}\right)\left(1-p_{11.13}\right)-p_{31.9} p_{13.8}\right\} \\
& \left.p_{03}\left\{p_{32.7}\left(1-p_{11.13}\right)+p_{3.19} p_{12.6}\right\}\right] \\
N_{3}^{s}=\mu_{3}^{\prime} & \left\{\left(1-p_{22.12}-p_{25}-p_{22.12,15}-p_{42.18} p_{24}\right)\right. \\
& {\left[\left(1-p_{11.13}\right) p_{03}+p_{13.8} p_{01}\right]+\left(p_{23.11}+p_{23.11,14}\right.} \\
& \left.\left.+p_{43.17} p_{24}\right)\left[1-p_{11.13}\right) p_{02}+p_{12.6} p_{01}\right] \\
& \left.+p_{24} p_{41.16}\left[p_{02} p_{13.8}-p_{03} p_{12.6}\right]\right\}
\end{aligned}
$$




$$
\begin{aligned}
N_{3}^{H R p}= & \mu_{4}^{\prime} p_{24}\left[p_{01}\left\{\left(1-p_{33.10}\right) p_{12.6}+p_{13.8} p_{32.7}\right\}\right. \\
& +p_{02}\left\{\left(1-p_{33.10}\right)\left(1-p_{11.13}\right)-p_{13.8} p_{31.9}\right\} \\
& \left.p_{03}\left\{\left(1-p_{11.13}\right) p_{32.7}+p_{12.6} p_{3.19}\right\}\right]
\end{aligned}
$$

and $D_{2}$ is already mentioned.

\section{Expected Number of Replacements of the Units}

Let, $R_{i}^{H}(t)$ and $R_{i}^{s}(t)$ the expected number of replacements of the failed hardware and software components by the server in $(0, t]$ given that the system entered the regenerative state $i$ at $t=0$.

The recursive relations for $R_{i}^{H}(t)$ and $R_{i}^{s}(t)$ are given as,

$$
\begin{aligned}
R_{i}^{H}(t) & =\sum_{j} Q_{i, j}^{(n)}(t) \&\left[\delta_{j}+R_{j}^{H}(t)\right], \\
R_{i}^{S}(t) & =\sum_{j} Q_{i, j}^{(n)}(t) \&\left[\delta_{j}+R_{j}^{S}(t)\right]
\end{aligned}
$$

Where, $j$ is any regenerative state to which the given regenerative state $i$ transits and $\delta_{j}=1$, if $j$ is the regenerative state where the server does job afresh, otherwise $\delta_{j}=0$.

Taking LT of relations and, solving for $\tilde{R}_{0}^{H}(s)$ and $\tilde{R}_{0}^{S}(s)$. The expected numbers of replacements per unit time to the hardware and software failures are respectively of given by,

$$
\begin{gathered}
R_{0}^{H}(\infty)=\lim _{s \rightarrow 0} s \tilde{R}_{0}^{H}(s)=\frac{N_{4}^{H}}{D_{2}} \text { and } \\
R_{0}^{S}(\infty)=\lim _{s \rightarrow 0} s \tilde{R}_{0}^{S}(s)=\frac{N_{4}^{S}}{D_{2}} \\
N_{4}^{H}=\left(p_{23.11,14}+p_{22.12,15}-p_{24}\right)\left\{p _ { 0 1 } \left[\left(1-p_{33.10}\right) p_{12.6}\right.\right. \\
\left.+p_{13.8} p_{32.7}\right]+p_{02}\left[\left(1-p_{11.13}\right)\left(1-p_{33.10}\right)-p_{31.9} p_{13.8}\right] \\
\left.+p_{03}\left[\left(1-p_{11.13}\right) p_{32.7}+p_{31.9} p_{12.6}\right]\right\} \\
N_{4}^{S}=\left\{\left(1-p_{25}-p_{22.12}-p_{22.12,15}-p_{42.18} p_{24}\right)\right. \\
{\left[\left(1-p_{11.13}\right) p_{03}+p_{13.8} p_{01}\right]+\left(p_{23.11}+p_{23.11,14}\right.} \\
\left.+p_{43.17} p_{24}\right)\left[\left(1-p_{11.13}\right) p_{02}+p_{12.6} p_{01}\right] \\
+p_{24} p_{41.16}\left(p_{13.8} p_{02}-p_{12.6} p_{03}\right)
\end{gathered}
$$

and $D_{2}$ is already mentioned.

\section{Expected Number of Visits by the Server}

Let, $N_{i}(t)$ be the expected number of visits by the server in $(0, t]$ given that the system entered the regenera- tive state i at $t=0$. The recursive relations for $N_{i}(t)$ are given as

$$
N_{i}(t)=\sum_{j} Q_{i, j}^{(n)}(t) \&\left[\delta_{j}+N_{j}(t)\right]
$$

where, $j$ is any regenerative state to which the given regenerative state $i$ transits and $\delta_{j}=1$, if $j$ is the regenerative state where the server does job afresh, otherwise $\delta_{j}=0$. Taking LT of relation (16) and solving for $\tilde{N}_{0}(s)$. The expected number of visit per unit time by the server are given by

$$
N_{0}(\infty)=\lim _{s \rightarrow 0} s \tilde{N}_{0}(s)=\frac{N_{5}}{D_{2}},
$$

Where,

$$
\begin{aligned}
N_{5}= & \left(1-p_{25}-p_{22.12}-p_{22.12,15}-p_{24} p_{42.18}\right)\left[\left(1-p_{11.13}\right)\right. \\
& \left.\left(1-p_{33.10}\right)-p_{13.8} p_{31.9}\right]-\left(p_{23.11}+p_{23.11 .14}+p_{24} p_{43.17}\right) \\
& {\left[\left(1-p_{11.13}\right) p_{32.7}+p_{31.9} p_{12.6}\right]-p_{24} p_{41.16}\left[p_{12.6}\right.} \\
& \left.\left(1-p_{33.10}\right)+p_{13.8} p_{32.7}\right]
\end{aligned}
$$

and

$D_{2}$ is already specified.

\section{Cost- Benefit Analysis}

The profit incurred to the system model in steady state can be obtained as

$$
\begin{aligned}
P= & K_{0} A_{0}-K_{1} B_{0}^{P}-K_{2} B_{0}^{R}-K_{3} B_{0}^{S}-K_{4} B_{0}^{H R_{P}} \\
& -K_{5} R_{0}^{H}-K_{6} R_{0}^{S}-K_{7} N_{0}
\end{aligned}
$$

$K_{0}=$ Revenue per unit up-time of the system

$K_{1}=$ Cost per unit time for which server is busy due preventive maintenance

$K_{2}=$ Cost per unit time for which server is busy due to hardware failure

$K_{3}=$ Cost per unit replacement of the failed software component

$K_{4}=$ Cost per unit replacement of the failed hardware component

$K_{5}=$ Cost per unit replacement of the failed hardware

$K_{6}=$ Cost per unit replacement of the failed software

$K_{7}=$ Cost per unit visit by the server

\section{Conclusion}

The numerical results for a particular case $g(t)=\theta e^{-\theta t}$, $h(t)=\beta e^{-\beta t}, f(t)=\alpha e^{-\alpha t}$ and $m(t)=\gamma e^{-\gamma t}$ are obtained to carry out the cost-benefit analysis of a computer system by giving priority to Preventive Maintenance over $\mathrm{h} / \mathrm{w}$ repair. Using these results the graphs for Mean 


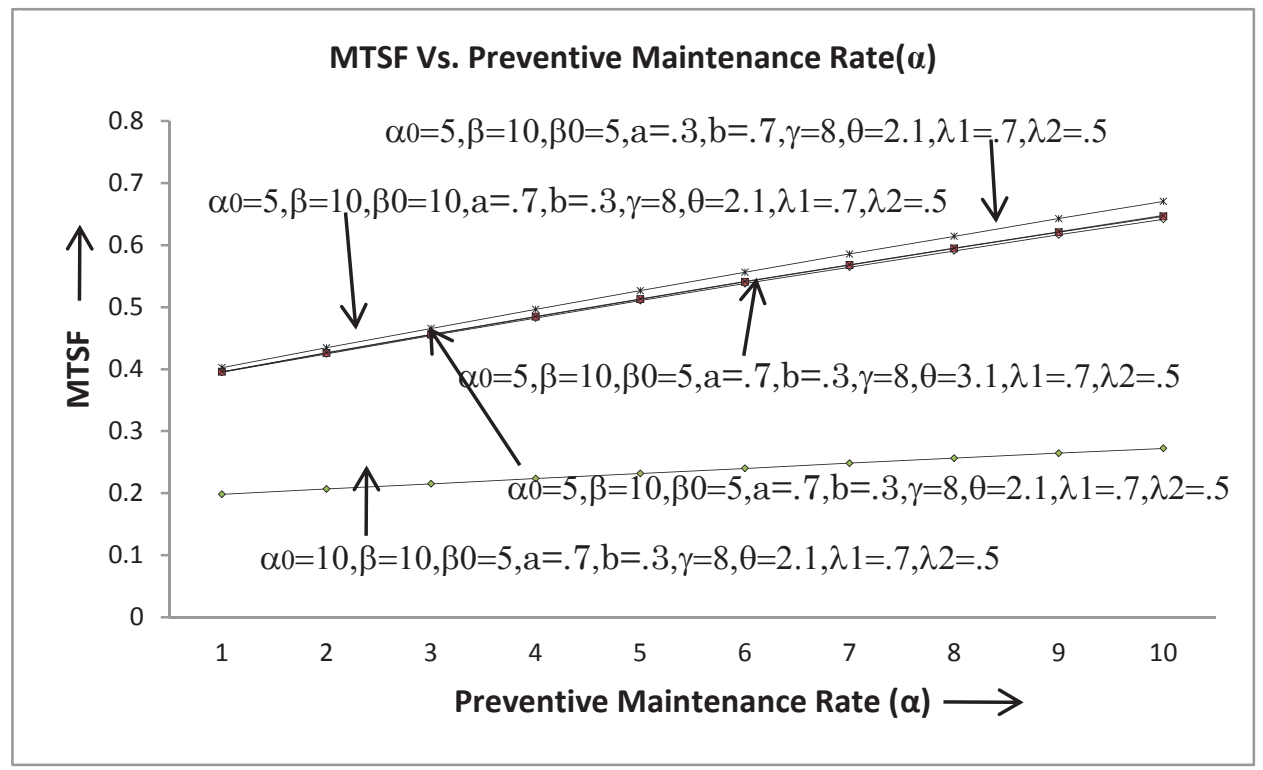

Figure 2. MTSF Vs. Preventive Maintenance Rate.

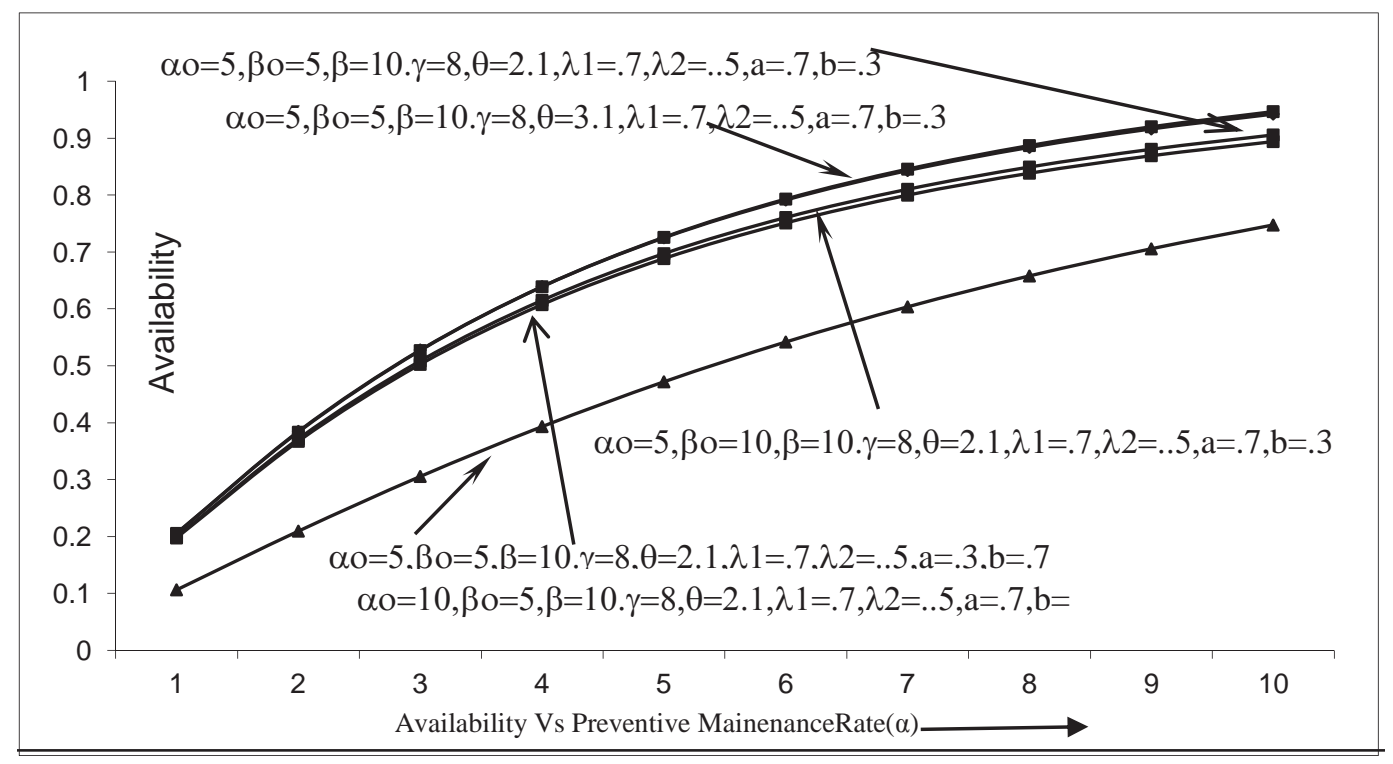

Figure 3. Availability Vs. Preventive Maintenance Rate.

Time to System Failure (MTSF), availability and profit are drawn with respect to Preventive Maintenance rate $(\alpha)$ for fixed values of other parameters including $a=0.7$ and $b=0.3$ as shown respectively in Figures 2, 3 and 4. From these figures, it is revealed that MTSF, Availability and profit increase with the increase of PM rate $(\alpha)$ and repair rate $(\theta)$. But the value of these measures decrease with the increase of Maximum Operation
Time $\left(\alpha_{0}\right)$. Again, if we increase the value of maximum constant rate of repair time $\left(\beta_{0}\right)$, the value of availability and profit are decreased while that of MTSF increases. Thus, a computer system can be made more reliable and profitable to use by:

(i) Conducting Preventive Maintenance after a Maximum Operation Time. 


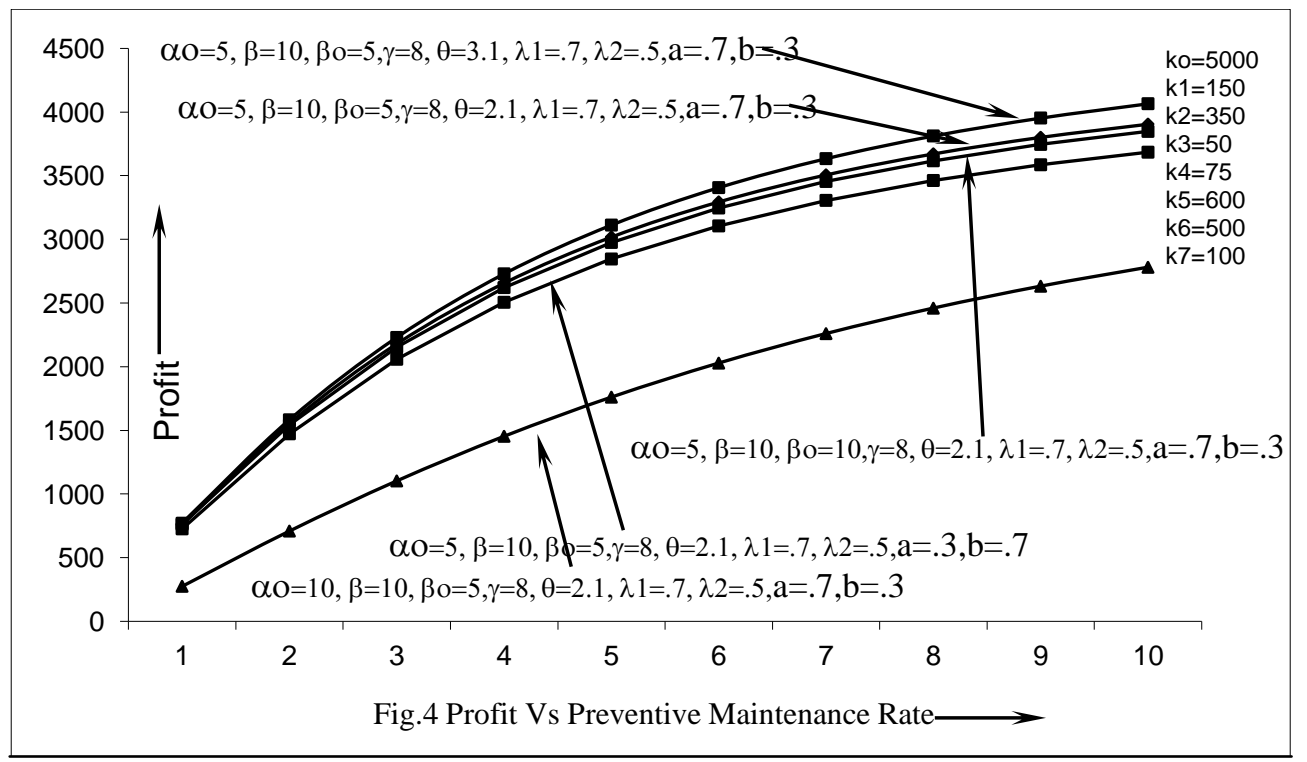

Figure 4. Profit Vs. Preventive Maintenance Rate

(ii) Giving priority to $\mathrm{PM}$ over $\mathrm{h} / \mathrm{w}$ repair.

(iii) Making replacement of $\mathrm{h} / \mathrm{w}$ by new one in case its repair server is not able to complete its repair with in a pre-specific time.

(iv) Making replacement of $\mathrm{s} / \mathrm{w}$ when it fails to execute the programs properly.

\section{Acknowledgement}

The authors are grateful to the reviewers for their valuable suggestions to improve the paper. The authors are also thankful to the Department of Science and Technology (DST), Govt. of India for providing financial assistance to carry out this work under the INSPIRE Fellowship.

\section{References}

1. Friedman M A, and Tran P (1992). Reliability Techniques for Combined Hardware/Software Systems, Proceedings, Annual Reliability and Maintainability Symposium, 1992, 290-293.

2. Welke S R, and Labib S W etal. (1995). Reliability Modeling of Hardware/ Software System, IEEE Transactions on Reliability, vol 44 (3), 413-418.

3. Malik S C, and Anand J (2010). Reliability and Economic Analysis of a Computer System with Independent Hardware and Software Failures, Bulletin of Pure and Applied Sciences, vol 29 E (1), 141-153.

4. Singh S K, and Agrafiotis G K (1995). Stochastic Analysis of a Two-Unit Cold Standby System Subject to Maximum Operation and Repair Time, Microelectronics Reliability, vol 35(12), 1489-1493. 